\title{
Relief and Psychological Contact to Information in Communication Support: A Study on Making Information Gestalt ${ }^{*}$
}

\author{
Yoshie Kiritani \\ Chiba University, Chiba, Japan
}

\begin{abstract}
This is a psychological study that propounds a user-centered theory for the design of communication support. The communication to be covered was that between experts as sender of information and non-experts as the receivers, the communication between university staff, and schoolteachers about an educational activity. The communication medium was a booklet to explain the activity. The introduction overviewed the psychological communication models and argued that the message that the receivers of information got was not always the same as one that the sender had had. Both of the sender and the receivers could have one's own information Gestalt. The first survey revealed teacher's current situation that they could have anxiety in the early communication with university's staff. Then, we set the design concept of the booklet as relief from the anxiety to produce a proper information Gestalt in the receivers. People who got catch up to worries would not receive the important information that the sender really wanted to inform them. The worries supposed in the present study were the support for children and the procedure of the class order. The main survey to working teachers revealed that the worries were accurately predicted; a typical situation in the Japanese public school was also shown. A strategy to reduce or make clear the vague concerns in the receivers of information would be new and effective in the support of communication between experts and non-experts. It means the reduction of psychological distance between the sender of information and the receivers.
\end{abstract}

Keywords: communication support, relief, information Gestalt, psychological contact, user-oriented design

\section{Introduction}

The present study gives psychological knowledge to communication design as a communication support study. We found that people as non-experts felt anxiety about the communication with experts. We designed a communication tool to release the anxiety and to get the communication smooth.

As user-centered design has been stressed, psychological findings have been widely referred by design practice. The present study picked up communication design that makes various media and aimed to give

\footnotetext{
* Acknowledgements: This work was supported by JSPS KAKENHI No. 23611007. The author expresses her appreciation for the participation in the survey to 10 teachers in Sagamihara city. The author is indebted to the members of ERCAZ who are now in Animal Life Solutions Co. Ltd; they use the booklet that is the final version of the prototype in the present study. The author also expresses her appreciation for the preparation of all of prototypes to the students who had engaged in the work, especially to Ms. Mizuki Yamazaki who drew illustration for the prototype in the present study and the final version.

Yoshie Kiritani, Ph.D., Department of Design Science, Chiba University.
} 
psychological advices for them. The media that we pay attention to were communication tools between experts and non-experts.

Communication between experts and non-experts sometimes has a problem because of the difference of their knowledge. For instance, instead of diffusion of informed consent in these days, the patients who usually do not have enough medical knowledge blindly follow doctors' advice and eventually receive unwilling treatment (Watanabe, 2012). The difference of knowledge occasionally brings emotional troubles as well as requires time-consuming communication. There is a need to bridge the divide between experts and non-experts.

The present study defines the attempts to bridge between experts and non-experts as communication support. Communication support involves concrete design works to help the communication. Although communication support is an activity of media design, the artifacts should be referred to psychological facts from the point of user-centered design. A series of design works have shown the discrepancy between them and proposed design works to solve problems (Abe, 2009; Kuwabara, 2010; Kiritani, Kuwabara, \& Tamagaki, 2012). All of these works were design proposals that aimed to raise the level of knowledge of non-experts. These works that did not have any theoretical background showed some effect and suggested the importance of the way of information provision to non-experts.

\section{Purpose of Study}

The purpose of the present study was to confirm the effect of elimination of anxiety of the information receivers for confirmation of the information Gestalt. The receivers of information were non-experts, while the sender of information was the expert in the present study. To effectively convey a message to people, a medium that could release from their worry was created and examined.

In this introduction, we firstly considered communication models and subsequently proposed a notion of information Gestalt to apply psychological facts to design artifacts. And the last, we explained the concrete situation of communication in the present study.

The first survey conducted the hypothesis that the elimination of anxiety of the receiver of information had a good effect. In the main survey, a prototype of the artifact to realize the hypothesis was made and examined.

\section{Communication Model and Information Gestalt}

Including Shannon and Weaver's model, some communication models have been proposed and they contain at least three elements: sender of information, its receiver, and message (Kawakami, 2009). The sender usually expects behavior change of the receivers. Kitamura (1985) introduced concepts of code and context in the communication model. The code means rules of coding and decoding of information and the context means the background of communication. If the rule of decoding of the information is different from the rule of coding, the receiver cannot get proper message from the sender of information. If the sender and the receiver do not share the same background, the communication cannot be established, neither. In the present study, the sender of information was an expert of the certain subject and the receivers were non-expert. The message was somewhat specialized information.

In the communication between experts and non-experts, the sender of information is expert and its receiver is non-expert. The sender provides the information as a message according to the own purpose. He/She has an intention of communication, because he/she has the information. Although in ordinary interpersonal communication, there is an alternation of the roles of sender and receiver, in the communication between experts and non-experts, the sender of information is definitely the expert, always set the code in the first stage 
of communication and of course has the own context. Moreover, it is not always true that the receivers want to get the information from the sender; they may be unwillingly involved in the communication in some cases, in the worst case.

For the receivers, the information as a message is provided from the external; the information is determined by the environment that they recognize. And when the receivers get the message, they interpret it according to their rule of decoding and their context. The information acquisition in this type of communication is substantially the same as the process of human perception and cognition. Thus, the author proposes a notion of information Gestalt.

Gestalt itself is a concept of Gestalt psychology and means an organized structure or its process (see Koffka, 1935/1988; Metzger, 1953/1968). It is an attribute of structure of the whole, not an aggregation of constituent elements. A melody is a good example of Gestalt; it is a whole that keeps the structure, even if all notes are transposed into another key and there is no part equal to the original melody. Gestalt can be regarded as figure of the behavioral environment that the livings grasp; the living organisms cut shapes out of its environment. The rules to derive Gestalt are known as figure-ground segregation and laws of grouping; the living organisms have the inside rules to make Gestalt from the environment (Kanizsa, 1980, 1991; Vicario, 1991).

If Gestalt is cut out of the environment, it is similar to the information as message in the communication. However, Gestalt is usually synonym of perception, while the information in the communication is recognized by the receivers. Perception is considered as lower process than cognition. Thus, the author uses the word of information Gestalt to distinguish them. Anyway, information Gestalt is the message contents received in the communication and is cut out of the receiver's behavioral environment. It means that the receivers produce information Gestalt according to their own rules that are not always the same as those of the sender of information. Remember that the sender provides the message using the own code and context. Thus, a problem occurs: the discrepancy between the message from the sender and the information Gestalt by the receivers.

To avoid the discrepancy, the sender of information should know how to emerge the information Gestalt in the receivers. Solving this problem is a communication support by the present study.

\section{The Communication Examined in the Present Study}

Communication concerning AAE (animal-assisted education) (Turner, 2009) was the case, as in the previous studies (Kuwabara, 2010; Kiritani et al., 2012; Kiritani, in press). AAE is one of activities of human-animal interaction and can be conducted by a regular schoolteacher with knowledge of the animal involved, but when conducted by a remedial (special education) teacher, a social pedagogue, or teacher working in a school, it is also considered to be therapeutic and a goal-oriented intervention.

In the present study, the sender of information is an expert of AAE, ERCAZ (the Laboratory of Effective Animals for Human Health, Educational Research Center for Anthrozoology) at Azabu University. ERCAZ had some AAE programs and one of them was a visiting class with dogs for elementary schools. According to ERCAZ, the class with dogs had following educational effects: experiential learning about creature or life, learning about rules and their application to the daily life, increase in motivation for lessons, and contribution to a sense of ease. Although it was difficult to show the quantified evidence of the educational effects, the schoolteachers participated in the class realized the educational effects and witnessed children's change. This class was not just an enjoyable event, but gave them a good chance to deeply think over the life. Kuwabara 
(2010) revealed that ERCAZ had over several years of experience in visiting class to elementary schools, like one of educational activities of Sagamihara city, Kanagawa prefecture. ERCAZ carefully arranged with the schoolteachers to match the visiting class to the requirements of each school.

The receivers of information in the present study were schoolteachers. They had no knowledge of AAE, so that they were non-experts in this situation. The communication medium was a booklet to explain the visiting class using dogs for schoolteachers. It would be useful to introduce the visiting class by ERCAZ for the schoolteachers of new entry.

At that time, ERCAZ had no documents of the overview or guidebook of the activity; at the meeting of early stage, ERCAZ might spend considerable time with schoolteachers. In ERCAZ, the necessary information was a kind of implicit knowledge and might be handed down from the person in charge to a new person like artisan skills. Another reason why ERCAZ had no written matters to use at the first meeting with a new client was that the staff could not afford to spend much time to produce them. Thus, the present study would make a booklet as a communication medium.

The booklet was firstly made according to ERCAZ's needs. Although the final version should be made to reflect the needs of the receiver of information, the prototypes of the booklet in early stages were forced to utilize ERCAZ's needs. It is the sender of information who has an intention to communicate. In fact, the prototypes in Kuwabara (2010), Kiritani et al. (2012), and Kiritani (in press) were based on the information from ERCAZ and made to realize the needs.

The needs were summarized as follows: (1) transmission of importance and significance of the class; (2) reducing the burden of the first meeting with newcomers; and (3) less load to provide information for the making of booklet. The first and the second needs were to be realized in the final version of prototype (see the left part of Figure 1).

\section{Needs of ERCAZ}

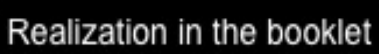

Needs of schoolteachers
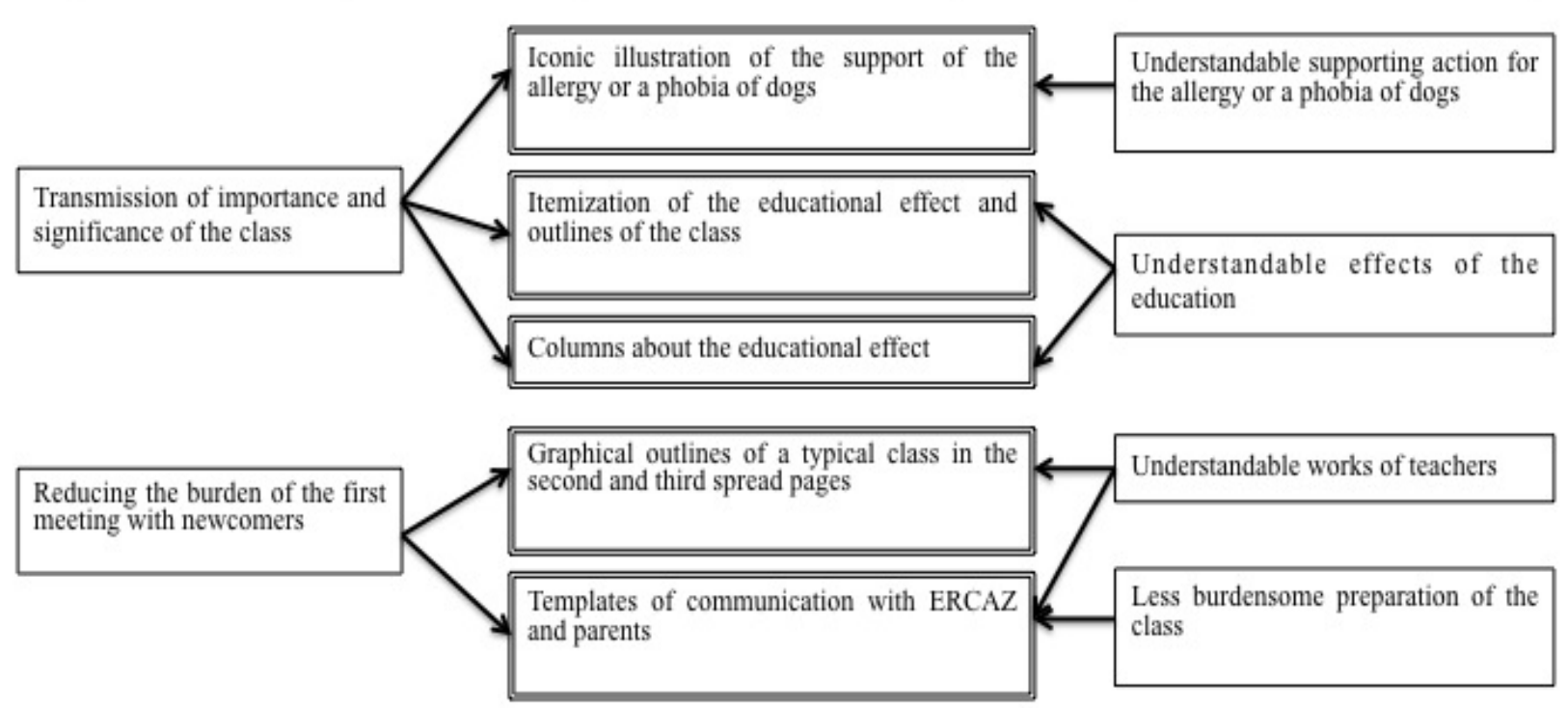

Figure 1. Relation among the needs of sender (ERCAZ), those of receivers (schoolteachers) and realization in the prototype. 
The third need would not be shaped in the prototype but related to its making. From the standpoint of ERCAZ, they were to give information to make the prototypes. To avoid the burden of this work, the minimum amount of meetings were required. There, vague questions were eliminated and concrete exemplification was shown. For instance, to make the part of FAQ, the researcher did not ask ERCAZ which questions they had had and what the answers were. Instead, the questions that the schoolteachers might have and their answers that ERCAZ might respond to were invented and proposed to ERCAZ. They just corrected the inappropriate expression.

\section{The First Survey to Know Needs of the Receivers of Information}

Kiritani et al. (2012) made a prediction about the needs of receivers of information. An evaluation method of simulated user called as acting evaluation revealed possible concerns of the schoolteachers; the most frequent concerns were children's safety and preparation for a phobia of dogs or the allergy, and, besides these opinions, dog's safety, hygiene, and consideration for other classes were reported.

The present study conducted interviews to the schoolteachers who had experienced ERCAZ's class and confirmed the reality of class and the needs of the receivers of information.

\section{Method}

Ten practicing teachers in two elementary schools in Sagamihara city, Kanagawa prefecture, Japan, answered interview questions. All of the teachers had actually conducted the class of ERCAZ for years, so that they answered retrospective questionnaire. Their age, gender, and assigned grader were shown in Table 1; five interviewees were in their $20 \mathrm{~s}$, four interviewees were in their $50 \mathrm{~s}$, and one interviewee was in her $60 \mathrm{~s}$; nine interviewees were female and one interviewee was male; seven interviewees taught the early graders, one interviewee taught the middle grader, and two interviewees taught a self-contained classroom.

A semi-structured interview was individually done. The researcher also asked them the current class of ERCAZ, but the main concern of the researcher was about their past experiences and event. It took about 30 minutes in the shortest interview and about 60 minutes in the longest one. The interviewees participated in the interview in the school during after school hour with permission of the principal or the vice-principal.

\section{Results}

Table 1 represents the answers to the principal questions.

As for concerns before the class execution (question 1), six of 10 teachers replied as children's allergy or a phobia of dogs. Two of them answered nothing and one of six teachers who pointed out the allergy problems said that she was in fact less concerned about them. Moreover, four of 10 teachers said that ERCAZ's explanation was actually very careful and that such worries proved to be groundless. As for minority opinions, parent's agreement, how to interact with dogs as an educational activity teacher's role, and relation between children in the special class and children in the normal class were reported. The most frequent concerns were almost the same as the results in the simulated experiment (Kiritani et al., 2012). One of particular opinion in the minority was the anxiety of a first-year teacher; she worried about all of activities in the school, so that she did not particularly worry about the ERCAZ's class.

It should be noted that the main source of information about the class (question 2) was the assistant principal or the principal who had already had experience of ERCAZ's class in their former schools. In other words, the top of school decided to conduct the class in the present school and other teachers prepared for it. 
There seemed to be no discussion to adopt the class; it was not open for discussion. Moreover, although the impressions before the class (question 3) were almost positive, most of teachers had thought that the class was like a fun event with animals.

Table 1

Results of Interviews of Schoolteachers in Sagamihara City

\begin{tabular}{|c|c|c|}
\hline Questions & \multicolumn{2}{|l|}{ Answers } \\
\hline \multirow{5}{*}{$\begin{array}{l}\text { 0) Age, gender, assigned } \\
\text { grader }\end{array}$} & (1) $50 \mathrm{~s}$, female, middle & (6) 20 s, female, early \\
\hline & (2) 20s, female, early & (7) 60 s, female, early \\
\hline & (3) 50s, female, special & (8) 20s, female, early \\
\hline & (4) 50 s, female, early & (9) 20s, female, early \\
\hline & (5) 20 s, male, early & (10) $50 \mathrm{~s}$, female, special \\
\hline \multirow{10}{*}{$\begin{array}{l}\text { 1) Concerns before the } \\
\text { execution of the class }\end{array}$} & \multicolumn{2}{|c|}{ (1) Hygiene and allergy of children. (Solved by ERCAZ's explanation.) } \\
\hline & \multicolumn{2}{|c|}{ (2) Nothing because of ERCAZ's careful explanation and leader teachers. } \\
\hline & \multicolumn{2}{|c|}{$\begin{array}{l}\text { (3) Nothing. (People loathing for dogs may have an image of bite. Although parents worry abou } \\
\text { allergy, they finally accept a situation because of children's behavior.) }\end{array}$} \\
\hline & \multicolumn{2}{|c|}{ (4) Allergy. (Solved by ERCAZ's explanation.) } \\
\hline & \multicolumn{2}{|c|}{ (5) Allergy. A phobia of dogs. But, not so serious concerns. (Hardly waiting to do the class.) } \\
\hline & \multicolumn{2}{|c|}{ (6) Parent's agreement. Allergy. } \\
\hline & \multirow{2}{*}{\multicolumn{2}{|c|}{$\begin{array}{l}\text { (7) How to interact with dogs, not as a play. Allergy. A phobia of dogs. (Solved by ERCAZ's } \\
\text { explanation.) } \\
\text { (8) How to teach. Role of teachers. (All of activities in the school are concerns. Anxious, because of } \\
\text { the first year.) }\end{array}$}} \\
\hline & & \\
\hline & \multicolumn{2}{|c|}{ (9) A phobia of dogs. Safety of dogs. } \\
\hline & \multicolumn{2}{|c|}{$\begin{array}{l}\text { (10) Nothing about the dogs. Relation between children in the special class and those in the norma } \\
\text { classes. }\end{array}$} \\
\hline \multirow{10}{*}{$\begin{array}{l}\text { 2) How to get the first } \\
\text { information about the } \\
\text { visiting class }\end{array}$} & \multicolumn{2}{|c|}{ (1) From the assistant principal who had experienced ERCAZ's activity in the former school. } \\
\hline & \multicolumn{2}{|c|}{ (2) From the assistant principal who had experienced ERCAZ's activity in the former school. } \\
\hline & \multicolumn{2}{|c|}{ (3) From the assistant principal who had experienced ERCAZ's activity in the former school. } \\
\hline & \multicolumn{2}{|c|}{ (4) From the principal. } \\
\hline & \multicolumn{2}{|c|}{ (5) Pictures in the board. From the leader teacher of the activity. } \\
\hline & \multicolumn{2}{|c|}{ (6) From other teachers, as a class of the first grader. } \\
\hline & \multicolumn{2}{|c|}{ (7) From the principal. } \\
\hline & \multicolumn{2}{|c|}{ (8) Pictures in the board. From other teachers. } \\
\hline & \multicolumn{2}{|c|}{ (9) From the principal who showed a kind of flyer. } \\
\hline & \multicolumn{2}{|c|}{ (10) From other teachers in the official meeting. } \\
\hline & \multicolumn{2}{|c|}{ (1) Healing or therapy in senior care homes. Seemingly good effect for children. } \\
\hline & \multicolumn{2}{|c|}{$\begin{array}{l}\text { (2) Ambiguous. Activities between children and visiting dogs. Seemingly interesting and fun. } \\
\text { Intelligent dogs. }\end{array}$} \\
\hline & \multicolumn{2}{|c|}{ (3) Seemingly fun. Good for children's feelings. Like an event, not educational one. } \\
\hline & \multicolumn{2}{|c|}{ (4) Hoped that children would be friends with dogs. } \\
\hline 3) Impressions of the class & \multicolumn{2}{|c|}{ (5) An event for children to play with dogs. Just fun, not educational. } \\
\hline & \multicolumn{2}{|c|}{ (6) A good occasion for children to interact with animals. } \\
\hline & \multicolumn{2}{|c|}{ (7) No image. (Stayed on the sidelines.) } \\
\hline & (8) Nothing particular. $A$ & for children to know animals. Just touching and seeing dogs. \\
\hline & (9) Like an interactive a & nall animals in zoo. Just touching. Many dogs would come. \\
\hline & (10) Interesting. & \\
\hline
\end{tabular}


Table 1 to be continued

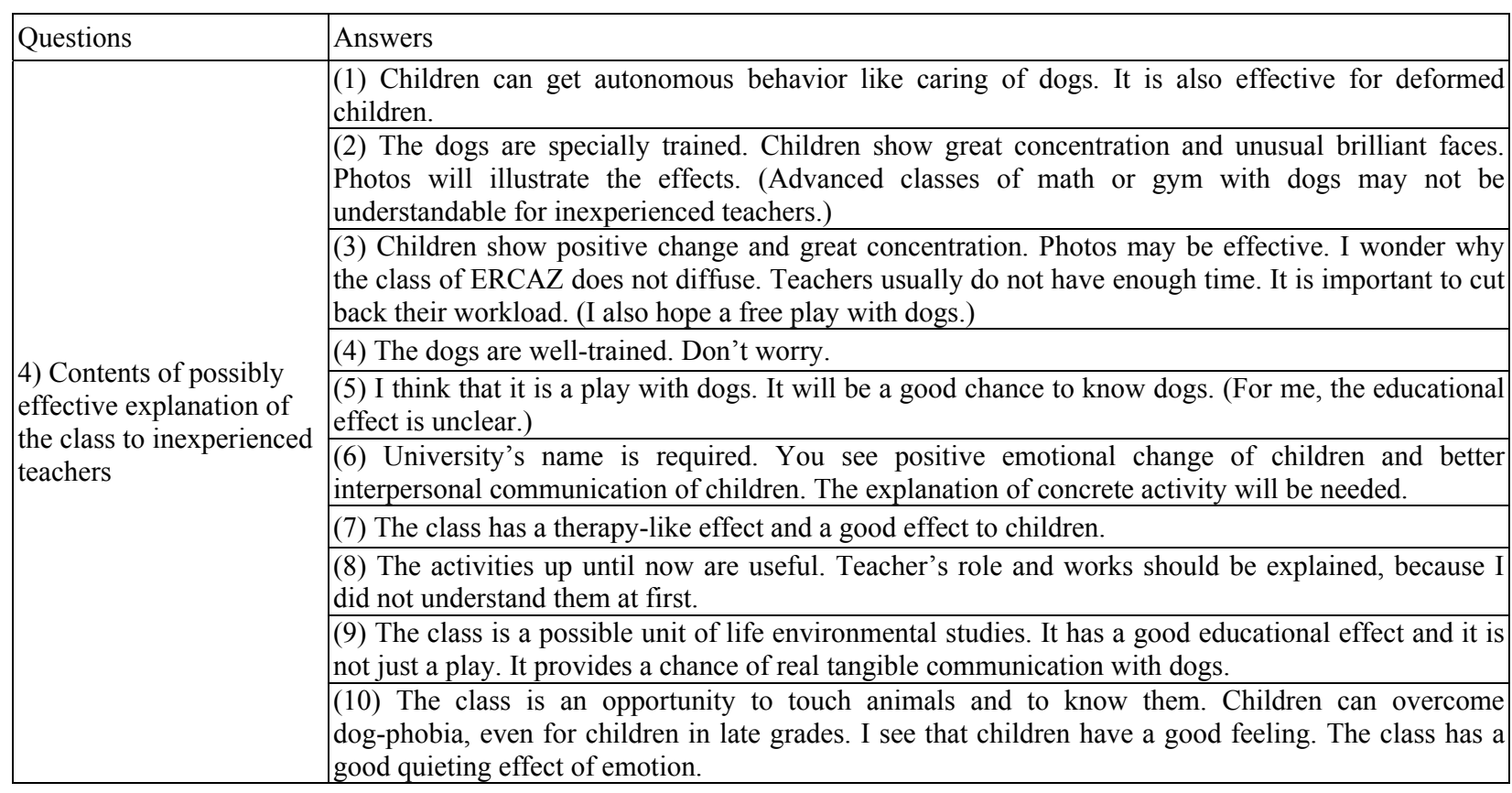

Note. ERCAZ means the Laboratory of Effective Animals for Human Health, Educational Research Center for Anthrozoology.

The teachers were also asked about the effective way of explanation to other inexperienced teachers (question 4). To inform positive effects for children was the most frequent answer; concrete examples of positive effects were autonomous behavior, great concentration, emotional change, better interpersonal communication, good quieting effect of emotion, and overcoming of the phobia. Although a young male teacher admitted a good chance to know dogs for children, he said that the concrete educational effect was unclear. A young female teacher said that the role and works of the schoolteachers in the class should be informed, because she had not understood at the beginning of the actual class. A fiftysomething teacher also pointed out the workload of teachers. Moreover, a veteran teacher pointed out that inexperienced teachers might not understand the advanced class of math or gym with dogs. Thus, the classes annually repeated by ERCAZ made the majority of teachers understand the good effect and trust ERCAZ's activity, but it was possible that some young teachers might not fully understand the significance and that there was a problem of the workload.

\section{Discussion}

The communication medium in the present study was a booklet that explained for the schoolteachers the way of request of the ERCAZ's class. In this class using specially trained dogs, all of children including allergy sufferers and children with a phobia of dogs could participate in. Using the booklet, the schoolteachers would understand outline of the class and ask ERCAZ for it without time and effort.

From the results of interviews, the present study presumed the receivers of information as subordinate teachers who had no decision right to adopt the class of ERCAZ but just prepared for it. They had good impression about it, however, they might not really understand the educational effect before the participation. It was possible that they were less-experienced teachers and nervous about their mission; some of them might unwillingly perform their duty, if they did not like dogs. The prime concern was the allergy or dog-phobia of children. The schoolteachers might be so busy to prepare the extra lesson of ERCAZ's class. 
Although the previous study properly predicted the concerns about the children who were not confortable with dogs, the burden of schoolteachers could not be detected. However, in this survey, only one young teacher mentioned her anxiety about events on the school calendar according to her poor experience, the opinion gave an important suggestion to the present study. The anxiety was an essential point of view for the user-oriented design. Usually, the medium in communication design is made to convey information that the sender wants to inform. However, if the receivers of information have some worries, the information will not be transmitted effectively. In the school, there are many matters for teachers to consider. To conduct smoothly the class of ERCAZ, the concerns, anxieties, or worries of the schoolteachers were regarded as important factors.

Thus, the needs of the receivers of information we summarized as follows: (1) understandable supporting action for the allergy or a phobia of dogs; (2) understandable effects of the education; (3) understandable works of teachers; and (4) less burdensome for teachers in the preparation (see the right part of Figure 1).

\section{Purpose of Main Survey}

The needs of the sender of information, ERCAZ, and those of the receivers of information, schoolteachers, led to the design concept of the prototype. The design concept was that the schoolteachers who had less time and less knowledge for the ERCAZ's activities could effectively get information about the big concerns. The point is to relieve concerns.

The information should be provided to solve the psychological problem of the receivers. The situation where the receivers of information are nervous about their mission is equal to the situation where there is a psychological distance between the receivers and the sender of information. Relief will reduce distance. The hypothesis was that the elimination of anxiety of the receiver of information had a good effect. The keyword for the design concept was relief.

However, the best situation would be that the schoolteachers completely relieve their concerns, the situation that, at least, they could clarify their questions or ideas for the first meeting was also acceptable from the point of the communication. It would be the worst case where they could not become conscious of their anxiety about the communication. Good communication could reduce the number of meetings; it was a big merit not only for the schoolteachers but also for ERCAZ's staff.

The relief would be realized in the prototype of booklet; each realization linked to the needs of ERCAZ and those of the schoolteachers (see Figure 1). The purpose of main survey was to examine the hypothesis and also to discuss its meaning from a point of the communication design. Interviews of small number of interviewees, but intensive, were conducted.

\section{Materials and Methods of Main Survey}

\section{Features of Prototype}

The prototype was a booklet that is a medium to explain the ERCAZ's class. The relief was realized in the prototype in the ways represented in the center part of Figure 1. Each realization linked to the needs of ERCAZ and those of the schoolteachers, as mentioned below.

Iconic illustration of children with white-and-red reversible cap (see Figure 2) was adopted to represent the support of the allergy or a phobia of dogs. In the class, ERCAZ used to use the cap to distinguish the children; if they did not like dogs, the children wore white cap; if they had an allergy to dogs, the children wore red cap; if they were comfortable with dogs, the children wore the cap around the neck but could wear the 
white cap when they got scared. In the prototype, the iconic illustrations of children wearing the cap repeatedly appeared to attract the attention of schoolteachers. This realization of the design concept related to the need of ERCAZ about the transmission of importance and significance of the class, because all of children could participate in the class regardless of their preference for dogs. Moreover, this realization related to the need of the schoolteachers about the understandable supporting action for these children.
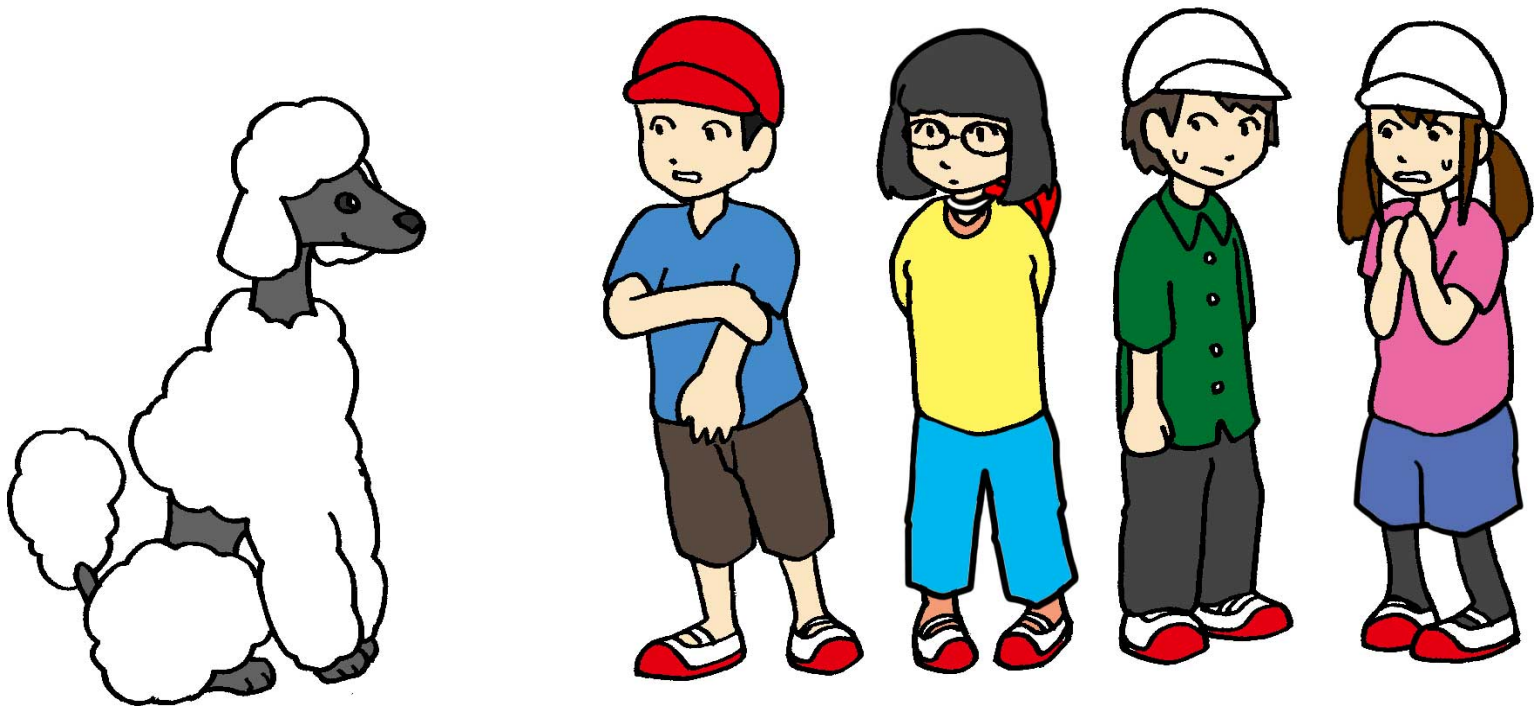

Figure 2. An example of the iconic illustration of children with white-and-red cap.

The first page of the prototype contained itemization of the educational effects and the outline of the class. This realization, once again, related to the need of ERCAZ about the transmission of importance and significance of the class and to the need of the schoolteachers about the understandable effects of education. Four columns about the educational effects according to the itemization in the first pages were also inserted in the prototype to describe the educational effects. This realization, as is obvious, related to needs of the schoolteachers about the understandable effects. These realizations had textual form but some photos showing happy smile of children or illustrations were accompanied.

On the other hand, graphical outlines were inserted in the second and third spread pages in the prototype. These graphics sequentially explained a typical class where trainers and supporters with dogs visited a school, explained how to greet dogs, encouraged children to communicate with dogs, explained the supporting action for children not good at dogs, and the role of schoolteacher in the class. The graphics appeared in the spread pages to attract attention of the schoolteachers. This realization related to the need of ERCAZ about the reducing burden of the first meeting and to those of schoolteachers about the understandable works.

Some templates, an arrangement sheet, a spreadsheet, and a questionnaire sheet, were inserted in the prototype. The schoolteachers could use them to communicate with ERCAZ or parents. The arrangement sheet was used to inform ERCAZ teacher's purpose and intention of the class. The spreadsheet could be used to inform ERCAZ the results of the questionnaire that could be conducted to parents using the questionnaire sheet. Since ERCAZ wanted to know the allergy to dogs and children's preference for dogs, the schoolteachers had to do a questionnaire. This realization of the design concept related to the need of ERCAZ about the reducing the burden of the first meeting and those of the schoolteachers about the understandable works and about the less burdensome preparation of the class. 
Appearance of the prototype was summarized as follows. The size was A4 and both side printing was adopted. The total page number was 50. It contained above-mentioned itemization of the educational effects and the outline of the class, graphical outlines of the class, index and six chapters: chapter 1 contents of the class, chapter 2 support for children who don't like dogs, chapter 3 preparation for the class, chapter 4 about the trained dogs, chapter 5 relevant material, and chapter 6 appendix. The appendix contained above-mentioned templates.

The order of information appeared in the prototype corresponded to the importance of information; the schoolteachers could get information that they wanted, if they read the prototype in order. The contents of the class were shown in a matrix in the chapter 2 and the process from the order to class was represented in a flow chart in the chapter 3; both of the graphic organizers used color coding system to easily indicate the relevant information that would appear later in the booklet. All of illustrations appeared near to text were linked to its contents.

\section{Participant}

Four working teachers of elementary schools in Tokyo or in Saitama prefecture in Japan participated in an interview. All of them had no previous knowledge about ERCAZ; they were recruited and rewarded by a research firm. Their features and the preference of dogs were represented in Table 2.

Table 2

Main Results of the Interviews for Working Schoolteachers

\begin{tabular}{|c|c|}
\hline Questions & Answers \\
\hline \multirow{4}{*}{$\begin{array}{l}\text { Age, gender, assigned } \\
\text { grader }\end{array}$} & (1) 27 , female, 3rd grader \\
\hline & (2) 58, male, 1 st grader \\
\hline & (3) 58 , female, 1 st grader \\
\hline & (4) 37 , female, 3rd grader \\
\hline \multirow{4}{*}{$\begin{array}{l}\text { A) What were your } \\
\text { worries about the class } \\
\text { during a time of } \\
\text { reading? }\end{array}$} & $\begin{array}{l}\text { (1) Support to children who have an allergy to dogs. I doubt that fall of children can participate in the } \\
\text { class. }\end{array}$ \\
\hline & $\begin{array}{l}\text { (2) Not-flexible schedule. Support to allergy suffers. (I hope that children who don't have pets at home to } \\
\text { touch animals.) }\end{array}$ \\
\hline & $\begin{array}{l}\text { (3) The max and min numbers of participants. Possibility of participation of parents. Possibility of } \\
\text { weekend class. }\end{array}$ \\
\hline & (4) Cost, schedule, and execution time \\
\hline \multirow{4}{*}{$\begin{array}{l}\text { B) Which pages did } \\
\text { you read to get the } \\
\text { overall image of class? }\end{array}$} & (1) 1.1 Class contents, and 5.2 Detailed information of class contents \\
\hline & $\begin{array}{l}\text { (2) Initial double track, a pie chart of time in } 1.2 \text { An example of class, and } 2.1 \text { Using red-and-white caps. } \\
\text { (Allergy is the biggest worry. Need for care.) }\end{array}$ \\
\hline & (3) Initial double track. \\
\hline & $\begin{array}{l}\text { (4) Photos of gymnastic hall in } 1.2 \text { An example of class. But I have already had the image because of the } \\
\text { experience in the former school. }\end{array}$ \\
\hline \multirow{3}{*}{$\begin{array}{l}\text { C) What do you think } \\
\text { of role of teacher? }\end{array}$} & $\begin{array}{l}\text { (2) Management of the time and place, management of children, and watching for children. I think that it } \\
\text { is difficult for us to make children touch dogs, so that I hope for the visiting lecturers to lead children in } \\
\text { the class. }\end{array}$ \\
\hline & (3) Management of children and translation of words of the visiting lecturers for children. \\
\hline & (4) Management of children and watching for children. \\
\hline \multirow{4}{*}{$\begin{array}{l}\text { D) Which pages did } \\
\text { you read to know the } \\
\text { detailed class? }\end{array}$} & (1) 5.2 Detailed information of class contents. \\
\hline & $\begin{array}{l}\text { (2) } 1 \text { Contents of class, } 3.7 \text { Meeting with ERCAZ, } 3.8 \text { Preliminary class, 3.9 Last preparation, } 3.10 \text { Role } \\
\text { of teacher in the class, and Column "expected effect 2, learning of rules". }\end{array}$ \\
\hline & (3) 5.2 Detailed information of class contents. \\
\hline & $\begin{array}{l}\text { (4) } 5.2 \text { Detailed information of class contents and } 5.3 \text { Examples of sequence. I did not read 1.1 Class } \\
\text { contents. }\end{array}$ \\
\hline
\end{tabular}


Table 2 to be continued

\begin{tabular}{|l}
\hline Questions \\
\hline $\begin{array}{l}\text { E) What did you } \\
\text { remember as clas }\end{array}$
\end{tabular} contents?

F) Which pages did you read to know the procedure of request?

G) How would you communicate the association?

H) Which pages did you read to know the educational effect?

I) What do you think of the educational effects?

J) Was the booklet effective to organize your thoughts?

\section{Answers}

(1) Guide dogs. The class seems to be applicable to petting rabbits in school in the 2nd grader. I did not understand the application for mathematics in 5.3. (I think that the booklet should contain concrete merits of the classes to persuade other teachers.)

(2) Petting dogs, real communication, dogs as animal, how to touch dogs and prohibited matter. (Many children have no pets like dog and cat and no experience of touching dog. Some children bring insects to school but can't care them. In the ordinary classes, there is no chance to touch the living animals. Children can care and have great attention to plants so that touching dogs will be able to evoke a feeling of affection.) (3) Petting dogs and care. I see a possibility of combination of some topics in a class. Indication of preference of dogs and allergy using white-and-red cap is good, because children themselves can tell their preference and change their opinion according to the feelings. (Warmth of dogs will remind in the children. Caring is a good experience. I hope a class containing many topics.)

(4) I cannot understand the application for mathematics in 5.3.

(1) 3.1 Flow up to the class. (It's very useful.)

(2) Almost all of 3. Preparation of class, arrangement sheet, and template of announcement for parents (I have already had image of the procedure from various experiences. Arrangement sheet and template are very useful. The flow of the day should be clear. I wonder if we have to inform the activity to the board of education. The class would be smoothly conducted, if it comes under an order from the principal. Teachers do not want to change existing events and introduce new ones.)

(3) 3.3 Announcement to the school and request for help, and 3.4 Confirmation of environment and preparation. Use of the arrangement sheet. (The template of announcement for parents is useful.)

(4) 3.3 Announcement to the school and request for help, 3.4 Confirmation of environment and preparation and 3.5 Contact with ERCAZ.

(1) Firstly, telephone. Fax would be the main line. I would not use postal nor electric mail.

(2) Telephone. (A simpler booklet to explain the lesson to other teachers would be needed. It is difficult to launch a new event or to change the existing flow of annual plan. Teachers are very busy.

(3) Telephone. I would use the fax to know the details. I would not use postal nor electric mail.

(4) Telephone and fax to know the cost and the schedule.

(1) Columns, especially Column 3 "Creating high motivation".

(2) 5.2. Detailed information of class contents and pictures in 1.2. An example of class.

(3) 4. About education support dogs, 5.2. Detailed information of class contents, an example of application to ordinal class in 5. Information, 5.2. Detailed information of class contents, and some columns.

(4) 5.2. Detailed information of class contents. (How many minutes does it take?).

(1) The class would teach rule compliance, understanding the procedure, and communication with dogs. It has some good effects to children. But I doubt the improvement in concentration.

(2) I think that the class has positive effect to children. It would give them an unusual experience. They would understand that dogs were warm, that they cast hair, that they lick something with their tongue, that they wag the tail, and so on. The children would understand the results, when the dogs licked their hand, for instance. The children would develop affection from the visiting dogs to other dogs and, finally, to human friends. It is important that the early graders have the class, so that the applied study like guide dogs in higher graders would be effective. I hope that the experience of petting dogs would develop to the understanding of the value of life.

(3) The class would have a positive effect to children. They would be able to find what they had slipped over before. (I really wish to ask the class to the association. I think that visiting classes naturally but intentionally make up for deficiencies in the ordinal classes. Our school likes visiting classes and often conducts them.)

(4) I think that the class has positive effect to children. (I have had an experience of the similar class in the former school.)

(1) It was effective to realize the questions.

(2) It was effective to understand the place and the action in the class, dogs' waste, the class image, the contents, communication to parents, and to-do lists.

(3) It was thankfully effective to organize ideas. The quality is so high that I have never seen it in similar activities. (I hope to use dogs' photos in the booklet, to remember the class for children. The photos would be useful, when the children write letters to dogs.)

(4) I was not effective, because I had already organized my ideas. I have enough experiences in many visiting classes. I would not use the booklet to explain the class to other teachers. For me, information about the place and about the arrangement of dogs and children in the class is just needed. 
Table 2 to be continued

\begin{tabular}{|c|c|}
\hline Questions & Answers \\
\hline \multirow{4}{*}{$\begin{array}{l}\text { K) Which pages were } \\
\text { effective to organize } \\
\text { your idea? }\end{array}$} & $\begin{array}{l}\text { (1) 5.2. Detailed information of class contents. I think that "1.1 Class contents as an overview" is not so } \\
\text { effective. }\end{array}$ \\
\hline & $\begin{array}{l}\text { (2) Initial double track, } 3.5 \text { Contact with ERCAZ, and 2. Support for children loathing for dogs or allergy } \\
\text { to dogs. (I think that the template for the communication to parents is useful.) }\end{array}$ \\
\hline & $\begin{array}{l}\text { (3) Dogs' photos in } 4 \text {. About education support dogs. (I think that the amount of visual information is } \\
\text { important.) }\end{array}$ \\
\hline & $\begin{array}{l}\text { (4) Nothing. I have already organized my ideas. (I think that the template for the communication to } \\
\text { parents is useful.) }\end{array}$ \\
\hline \multirow{4}{*}{$\begin{array}{l}\text { L) What are negative } \\
\text { aspects in the booklet? }\end{array}$} & $\begin{array}{l}\text { (1) Nothing particular. But I want a template to persuade parents and the feedback from the teachers who } \\
\text { have already conducted the classes. }\end{array}$ \\
\hline & $\begin{array}{l}\text { (2) Nothing particular. But I do not need dogs' photos. I want photos in the classes and the concrete } \\
\text { arrangement of children and dogs in the class to understand the flow. The volume of booklet is too heavy. } \\
\text { (The order of information presented in the booklet is good.) }\end{array}$ \\
\hline & $\begin{array}{l}\text { (3) Nothing particular. But I did not understand the meaning of "topic" in 1.1 Class contents. The volume } \\
\text { of booklet is too heavy. I found some disgusting expression in the columns. I want an explanation of the } \\
\text { outline of class for parents. }\end{array}$ \\
\hline & $\begin{array}{l}\text { (4) Nothing particular. But the volume of booklet is too heavy. I think that illustrations for decoration are } \\
\text { not necessary. I want a more compact booklet. Small amount of information is enough: two samples of } \\
90 \text {-minute class, the place, countermeasures in children loathing for dogs or allergy to dogs and the } \\
\text { preparation. I cannot understand why children hook caps in the neck, because this action is dangerous (it } \\
\text { is forbidden in my school). }\end{array}$ \\
\hline \multirow{4}{*}{$\begin{array}{l}\text { M) What did you think } \\
\text { of the classes (by } \\
\text { ERCAZ)? }\end{array}$} & $\begin{array}{l}\text { (1) They are good for children. But it would be difficult to persuade other teachers. (Teachers don't want } \\
\text { to change the current style.) }\end{array}$ \\
\hline & $\begin{array}{l}\text { (2) They are realizable as classes of the life environmental studies in the } 1 \text { st grader. It seems to be } \\
\text { difficult to get the place to gather all of children in the grader at one time. }\end{array}$ \\
\hline & $\begin{array}{l}\text { (3) They are realistic as classes of the life environmental studies. I have a good impression. The purpose } \\
\text { is reliable. The use of cap is a well thought-out strategy. But a topic of } 40 \text { minutes is too long. (We } \\
\text { welcome visiting classes.) }\end{array}$ \\
\hline & $\begin{array}{l}\text { (4) I think that the classes are careful and that the booklet is well constructed. They are realizable, } \\
\text { especially as a class of Japanese in the } 3 \text { rd grader. But it is needed to reconstruct of time from } 60 \text { minutes } \\
\text { to } 45 \text { or } 90 \text { minutes according to the real time schedule in the elementary schools. }\end{array}$ \\
\hline \multirow{4}{*}{ N) Do you like dogs? } & (1) I do not like dogs. \\
\hline & (2) I had had a dog in my childhood. Now I have two cats. \\
\hline & (3) I like dogs and animals very much. \\
\hline & (4) I do not say that I like dogs nor do not. But I am not afraid of dogs. \\
\hline
\end{tabular}
Notes. The numbers in the answer column correspond to the interviewees described in the participant. The descriptions in parenthesis were comments not directly corresponded to the current question. ERCAZ means the Laboratory of Effective Animals for Human Health, Educational Research Center for Anthrozoology.

For a comparison, a female $31 \mathrm{Y} / \mathrm{O}$ teacher who worked in a private school in Tokyo was also interviewed; her assigned grader was the 4 th.

\section{Procedure}

Before the interview, each interviewee had received the prototype of booklet and read it according to the instruction. The instruction set a situation and required the interviewees to image it. There, the school of the interviewees decided to introduce the visiting class with dogs of ERCAZ to the graders of the interviewees and they were appointed as the liaison between ERCAZ and the school. The interviewees should have read the prototype to do a meeting with ERCAZ and made themselves clear about the issues of the class. However, in the prototype the names of ERCAZ, that of Azabu University and their contact information were masked to avoid a real contact between the interviewees and ERCAZ. They just informed that a certain association in a certain veterinary university hosted the class. 
The interviewees decided the place and the date of interview. It generally took about one hour in a downtown cafe. Although two staffs of the research firm shared a table with the researcher and the interviewee, the researcher mainly queried with the interviewee. A semi-structured interview was done. Most of questions were set to confirm following points: (1) whether the concerns of teachers were the same as the researcher supposed; (2) whether the concerns were relieved after reading the prototype; and (3) whether the information of outline of the class, its procedure of order, and the educational effects were properly understood. The design problems in the prototype were also asked.

To clarify the significance of the results from above-mentioned four interviewees, a schoolteacher in a private school was also interviewed in the same way.

\section{Results and Discussion}

The required time to read the prototype was about one hour for No. 1,2, and 3 interviewees, and less than 20 minutes for No. 4 interviewee. Main questions and their summarized answers were shown in Table 2.

\section{Concerns of the Teachers}

The present study had set the concerns of schoolteachers about the class of ERCAZ as the supporting action for an allergy or for a phobia of dogs and the bother about the procedure. The related question was Question A.

No. 1 and No. 2 interviewees pointed out the supporting action and all of interviewees pointed out were about the procedure. Both of them concern that the present study predicted was pointed out; especially the procedure was more important than the supporting action. The attitude and facial expression during the interview can also give us important information about the priority.

No. 1 interviewee doubted if all of children including children who were not confortable with dogs could participate in the class. According to the question N, this interviewee herself did not like dogs. On the other hand, her answer in question $\mathrm{E}$ also indicated that she worried about the way to persuade other teachers to realize the class. It reflects that her concern was the procedure of the class.

The bigger concern of No. 2 interviewee was about the procedure. He also worried about the schedule that was relevant to the procedure. He pointed out that the school schedule had been fixed at the end of the previous school year and that it was very difficult to insert a new event. He indicated the supporting action as the second concern, but he made another comment; he wanted children who had no pets at home to communicate with animals. Question $\mathrm{M}$ indicates that he liked dogs. The long comment on the question I, views about the educational effect of the class, represents that he understood its positive effect. It suffices to say that he worried about the support for the special children in general terms. His main concern was the procedure.

Indication of No. 3 and that of No. 4 interviewees presupposed the execution of the class; they thought about how to do it. But the nuance of their reports was not negative too much. Especially, No. 3 interviewee positively commented, too.

In short, concerns of the interviewees were summarized as two points: the procedure of the class and the support for the children who were not confortable with dogs. Thus, the concerns supposed in the present study were regarded as appropriate.

For reference, No. 2, 3, and 4 interviewees were asked about the role of schoolteacher in the class (question C). All of them replied that it was the management of children. In fact, it was the correct answer. Thus, these interviewees properly read the role in the class from the prototype. 


\section{Relief From the Concerns}

It is ideal that the prototype could solve the concerns of the interviewees. At least, it is meaningful that the prototype helped the users to organize their thoughts for the first meeting with ERCAZ. The role of the prototype was the relief from the concerns or their consciousness, because the consciousness would lead to clear questions that would reduce the required time for the first meeting.

Question J confirmed whether the interviewees recognized the effect of the prototype to organize their thoughts for the meeting with ERCAZ. No. 1, 2, and 3 interviewees said "Effective"; especially No. 3 interviewee praised it a great deal and said that she had never seen any material so careful before. The effect was confirmed.

However, only one interviewee, No. 4, did not admit the effect; she had already organized her thoughts from her previous experiences about other visiting classes and did not particularly require the overall information about ERCAZ's activity.

The parts of the prototype useful to organize the thoughts were showed in the answers of question $\mathrm{K}$. The useful parts were different among the interviewees according to their previous experiences as schoolteacher.

For No. 1 interviewee who was a younger teacher, the detailed information about the classes was useful, but their overview table appeared in early pages was not so effective for her. No. 2 interviewee, a veteran teacher, referred to the initial double track, information about the contact with ERCAZ, and the support for dog-phobia and allergy as effective and the template to use for communication to the parents as useful. The answers of No. 3 interviewee, a veteran teacher, seem to be irrelevant for the question, but the answer to question J can complete that of question K. She wanted to use dog's photos in a following lesson; she invented a class where the children wrote a letter of thank to dogs and thought that the photos in the prototype were useful to remember the dogs. Thus, she fully understood the important points of the ERCAZ's class and considered the further lesson after the class. No. 4 interviewee who had already organized her ideas and did not need the prototype also admitted the usefulness of the template to communicate the parents.

Only No. 2 interviewee definitely solved his concern about the support for children by the prototype. However, the answers to questions $\mathrm{J}$ and $\mathrm{K}$ revealed that the prototype was helpful to understand the overview, the procedure of order and important points of the ERCAZ's class. The parts needed by schoolteachers were various and the prototype could afford the needed information.

\section{Information Received From the Prototype}

Which part did the interviewees read to get proper information? The answers were useful to check the composition of the prototype. Questions B, D, E, F, H, and I were related in this point.

Question B was done to know which part the interviewees read to grasp the overall image of the class. The double track in early pages to graphically inform the overview of the class was one of contents that the prototype prepared for the users to get overall image of the class. This content was actually effective. No. 2 and No. 3 interviewees who were veteran teachers confirmed the effect as answers of question B; they easily understood the image of class from the illustrations in the prototype. Other comments revealed the effect of another contents of the prototype.

No. 2 interviewee also referred to the way of the support for dog-phobia or allergy. The method was an iconic illustration of children with white-and-red reversible cap. No. 2 interviewee said that the illustration repeatedly appeared in the prototype was impressive. The illustration was repeated to be memorable, so that it was effective. 
No. 1 interviewee, a young teacher, said that the class contents were useful; she might not clearly imagine the class itself and try to understand what it was. The class contents were inserted to give more concrete information of the class, which would be also useful to get the overall image of the class for less-experienced teachers.

On the other hand, the schoolteacher who had already had the image of the class required another information. No. 4 interviewee said that a real photo inserted in the prototype was useful; she might want to get information to confirm her idea.

Question D was about the pages read to know the detailed information of the class. The outline of class contents as a matrix in the 1 st chapter and the detailed information of the class appeared in the 5 th chapter were prepared in the prototype to give the information. The answers to question D revealed the effect of the 5th chapter. No. 1, 3, and 4 interviewees read this chapter. Incidentally, question E was a question to confirm if the interviewees really understood the class contents; it revealed that all of the interviewees did it.

Only No. 2 interviewee read mainly the 3rd chapter that was about the preparation for the class; he might imagine the class from its outline and consider the procedure of preparation as more important.

Question F was set to know which pages the interviewees read to get information about the procedure of request. The prototype contained the information in the 3rd chapter. All of interviewees read this chapter, as expected. The flow up to the class, and templates were evaluated as useful. No. 2 interviewee pointed out the needs of information to persuade the local board of education and the difficulty of introduction of a new event.

Question $\mathrm{H}$ was about the educational effects of the class. The prototype contained an itemization in the first page and four separate short columns about the educational effects. No. 1 and 3 interviewees read the columns. But, the interviewees got the information from other contents of the prototype.

Three interviewees except for No. 1 interviewee got the information mainly from the 5 th chapter that explained the detailed contents of the class. The schoolteachers who had a certain level of experience might easily understand the educational effects of the class and not require much information. To confirm this point, question I was done. All of interviewees admitted the positive educational effects in the class. Especially, No. 2 and 3 interviewees told it enthusiastically, also in questions A and E.

For reference, question $\mathrm{M}$ asked about the impression of the class by ERCAZ. All of interviewees had positive impressions, like a good effect for children or a realizable activity.

On the other hand, also to this question, No. 1 interviewee pointed out the difficulty to introduce a new event in the school; she might acknowledge the benefits but worry about the introduction or a change in the routine work. Thus, again the burden of schoolteachers was shown.

\section{Comparison With an Interview to a Teacher of a Private School}

As mentioned above, all of four interviewees admitted the positive effect of the class by ERCAZ. The results of interviews seemed to be almost favorable to the present study. However, there was a difference arisen from the experience, their concerns were mainly the support for children who disliked dogs or had an allergy or the concrete procedure to conduct the class including the preparation. The prototype had expected these concerns and taken countermeasures.

The expecting users of the booklet realized as the prototype in the present study were schoolteachers of public school, since ERCAZ had conducted the class in public schools. The concepts were set according to this fact. Thus, the prototype was for teachers in public schools. In fact, the interviewees understood the 
experimental instruction that the principal had already decided to conduct the class and asked her/him to be a manager; they read the prototype to execute the program. To clarify the limit of effect of the prototype, a teacher from a private school was also interviewed.

As a result, this private school teacher had rather bad impression about the ERCAZ's class and said that she checked the prototype from a severe view of the private schools. Her indication was that the intention, purpose or goal of education was unclear. She personally liked dogs but doubted the effect of dogs' visit to schools, because many pupils in her school had some pets at home in the first place. She felt that the class of ERCAZ would become an amusing event to children, not a chance for education. She said that the most important thing was the conformation to the educational policy of the school and that the support for allergy was secondary concern. According to her, all of pupils concentrated on the lesson at the beginning and there was no need to ask a help to dogs. She clearly said that she would refuse the class, even if the principle asked her to introduce it. She also doubted the organizer of the class, ERCAZ, and she wanted to know the licentiate; in the prototype, to avoid the later troubles, all of information to identify ERCAZ was withheld.

There were great different points between public schools and private schools. Firstly, it was the family environment of children. Not only the interviews to above-mentioned four interviewees but also those in the prior section revealed that there were few children who had pets at home in the public school. The teachers in the public school admitted the effect of animals, even if children just touched and felt them. However, in the private schools, children have some living animals at home, so that the schoolteachers may want a superior effect of education.

Secondly, the difference appears in the acceptance of a mission. The private school teacher said that she would refuse the mission to introduce the class, even if the president asked her. But, all of interviewees who were teachers of public schools did not say such a thing, accepted the setting and answered to the questions. Moreover, the private school teacher doubted whether ERCAZ was a real certificated organizer, but the four interviewees did not get suspicious at all. Thus, the four interviewees tended to follow the mission that the principal ordered without question.

The comparison of interview between the public schoolteachers and the private schoolteacher revealed the peculiarity of the public schools. The teachers in the public school seemed to execute the mission, if they were asked for. For them, the information to conduct the mission was more important than the educational effects. The prototype resolved this problem and would be for the teachers in public school.

\section{Visual or Structural Problems of the Prototype}

Question L asked the interviewees about the bad aspects of design in the prototype in a straight manner. Although the first answer of all interviewees was "nothing particular", intensive following questions revealed their real opinions. Heavy volume was indicated by three of them. No. 1 interviewee complained about the lack of a template to persuade parents and the less information of feedback from the teachers who had already done the class. This comment represents her anxiety for the class. No. 2 interviewee indicated the unnecessity of dogs' photos but the necessity of photos to show the concrete arrangement of dogs and children in the class. This opinion reflects the need of information to help him on class execution. The indication by No. 3 interviewee was mainly about the literal expression. No. 4 interviewee said that the compactness was the most important and the illustration or decoration was not needed.

As a positive aspect, No. 2 interviewee gave a good account about the order of information in the 
prototype. Moreover, question G showed the adequacy of medium; all of interviewees answered that they would use telephone or fax to communicate with ERCAZ and two of them said that they would not use e-mail. Thus, the prototype that was a printed booklet and not an electronic file was more proper in the current situation of public schools.

\section{Discussion}

The present study had the hypothesis that the elimination of anxiety of the receivers of information had a good effect to the communication. The design concept of prototype that was a communication medium was the elimination of anxiety or the release from it. The anxiety or concern of the receivers of information that were the schoolteachers in the present study was considered as the support for the children who did not like dogs or had an allergy or as the burden of procedure for the class order. Although it was unclear that the present study really alleviated the worries, the interviews revealed that the supposition of the worries of schoolteachers was appropriate.

The comparison between the public school teachers and the private school teacher revealed the difference of concern. That of the formers who would be the real users was about the procedure. Although the support for the children who were not confortable with dogs was also a big concern, this countermeasure can be regarded as a part of the procedure of the class. The teachers in public schools seem to trust its educational effect. The order of priority was completely different from that of the private school teacher; she insisted that the most important things was the educational effect. Interestingly, she seemed to trust the support for the children who did not like dogs, because she believed that the organization like ERCAZ which conducted such a class definitely had countermeasures for the dog-phobia or the allergy.

The teachers in public schools had a heavy workload in the daily routine and felt stress about the introduction of new events. The interviews to No. 1 and No. 2 interviewees revealed this tendency. They did not want to change the familiar procedure. Although No. 1 interviewee was a young teacher and No. 2 interviewee was a veteran teacher, they had the similar concern. For them, the mission from the top should be done; they wished to conduct it in an effortless way. A reduction in the burden of the daily work is an important matter for the public school teachers.

This view was confirmed also by a recent research of OECD (2014). It reveled that Japanese teachers had the longest working hours in 34 countries and regions; on the other hand, Japanese teachers evaluated themselves lower than those in the other countries and regions did. Also an article of NHK (2014) reported how Japanese teachers were busy and could not spend much time on the education. To help them, the support for reducing the burden of the daily work is a key.

The prototype in the present study provided the information that could reduce the burden to conduct the special class. In the communication support that aims at conforming the receivers' information Gestalt to that of the sender, it is the point to grasp what is the burden for the receivers of information. The burden can inhibit information acquisition.

The present study regards the information provision based on the dislike of the receivers. Usually, the information provided in the communication design is based on what the sender wants to give. It is the appeal by the sender. However, it is not true that what the sender wants to appeal is the same as the receivers want to know. If they are subjected to stress, they may not properly get information. The prototype in the present study emphasized the information that the receivers worried about and facilitated recovery from the anxiety. In the 
case of this study, the concerns of the receiver were derived from the experiment and the survey in the first survey and were realized in the prototype as a design concept. And the interviews of the main survey revealed that this method was effective.

Although the number of participants was rather small, the present study illustrated the negative emotion of the schoolteachers. They were not effusive in their praise for the prototype.

Then, let us consider the significance of the design concept in the present study from the point of the communication model.

The design concept was focusing on the release from the burden for the receivers of information. This attitude relates to the contact of information. Referring to Roman Jakobson, Akase (2013) pointed out six factors to establish communication: addresser (sender of information), addressee (receiver of information), message, context, code and contact. Although the former five factors were explained in the introduction, the last factor, contact, was not. It means not only physical pathway but also psychological connection. Although the code is usually regarded as the most important factor in the design, the contact, especially psychological one, is a fundamental. If the receivers do not contact the sender or the message, the information will not reach to the receivers. If they have a distance from the message, the supporter of communication should get rid of the distance.

In the present study, the approach to relief from the anxiety means reducing the psychological distance between the sender and the receivers of information. It is the first step for smooth communication.

\section{Conclusion}

The present study proposed hypothesis that the elimination of anxiety of receivers was effective for creation of information Gestalt to get the smooth communication of experts and non-experts. It was the design concept revealed from Kiritani et al. (2012) and the first survey. The needs of the receivers of information were summarized as the elimination of anxiety. The interviews confirmed the effectiveness of the design concept and the hypothesis. This attitude meant reducing the psychological distance between the sender and the receivers. The approach to pay attention to the psychological contact is important for the communication design.

To find the concern of the receivers is a mission for the supporter of communication, that is, the designer of communication support. Since the senders of information have the aim for the communication, they might not notice the receivers' real state of mind. The third party can operate in a neutral way.

Further studies would be required to confirm the effect of focusing the concerns of the receivers or their elimination, because the present study is just a case study.

In the last place, as to the design of a booklet to explain ERCAZ's activity, there is a room for improvement, though the prototype had fulfilled a role to inform basic and important information. To reduce the psychological distance more, the booklet should be in a style that the schoolteachers would want to read it. Since they do not have enough time to prepare the classes, additional work should be minimized. The volume of the prototype in the present study was rather heavy, because the prototype contained all of information that ERCAZ wanted to provide. To keep the concept, the reduction of information will be inevitable in the final version. It also means the narrowing down of users; the booklet should be for the schoolteachers who want to know the procedure of class. The educational effect will not be put, because the schoolteachers will really understand it during the class. 


\section{References}

Abe, S. (2009). Communication support between clients and designers to produce motion picture (Master thesis of Graduate School of Engineering, Chiba University). (in Japanese)

Akase, T. (2013). Signology for public spaces. Kajima Publishing: Tokyo. (in Japanese)

Kanizsa, G. (1980). Grammatica del vedere. Il Murino: Bologna. (in Italian)

Kanizsa, G. (1991). Vedere e pensare. Il Murino: Bologna. (in Italian)

Kawakami, Y. (2009). Interpersonal communication. In H. Hori (Sup. Ed.) New version, social psychology (pp. 164-185). Fukumura Publishing: Tokyo. (in Japanese)

Kiritani, Y., Kuwabara, S., \& Tamagaki, Y. (2012). A test of acting evaluation to assess a proposal of communication support study. Bulletin of Japanese Society for the Science of Design, 59(1), 31-38.

Kiritani, Y. (in press). Acting evaluation as an assessment method using participant's imagination. Bulletin of Japanese Society for the Science of Design.

Kitamura, H. (1985). Signology in TV and media. Yuseido Kobunsha: Tokyo. (in Japanese)

Koffka, W. (1935). Priciples of Gestalt psychology. Routledge \& Kegan Paul Ltd.: London.

Kuwabara, S. (2010). A proposal of kit about animal assisted education of for schoolteachers. Graduation work of Department of Design, Chiba University. (in Japanese)

Metzger, W. (1953). Gesetze des Sehens. Waldmar Kramer \& Co.: Frankfurt. (in German)

NHK. (2014). http://www3.nhk.or.jp/news/web_tokushu/2014_0801.html (in Japanese)

OECD. (2014). http://www.nier.go.jp/kenkyukikaku/talis/ (in Japanese)

Turner, D. C. (2009). Anthrozoology—now past its juvenile years! Change by Anthrozoology in Japan, 1, 4-7.

Vicario, G. B. (1991). Psicologia generale. Editori Laterza: Roma. (in Italian)

Watanabe, Y. (2012). Don't rush into a surgery, when your doctor informed you that you have got a breast cancer. Komichi Shobou: Tokyo. (in Japanese) 